Editorial

\title{
Advances of Theranostics of 2019-nCoV
}

\author{
Daxiang Cui ${ }^{1,2}$ \\ ${ }^{1}$ National Engineering Research Center for Nanotechnology, 28 East Jiangchuan Road, Shanghai 200241, China. \\ ${ }^{2}$ Institute of Nano Biomedicine and Engineering, Shanghai Engineering Research Centre for Intelligent Diagnosis and Treatment \\ Instrument, Department of Instrument Science and Engineering, School of Electronic Information and Electrical Engineering, \\ Shanghai Jiao Tong University, 800 Dongchuan Road, Shanghai 200240, China.
}

Corresponding author. E-mail: dxcui@sjtu.edu.cn

Published: Dec. 8, 2020

Citation: Daxiang Cui, Advances of Theranostics of 2019-nCoV. Nano Biomed. Eng., 2020, 12(4): 358-359.

DOI: 10.5101/nbe.v12i4.p358-359.

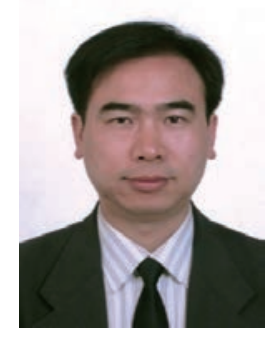

Daxiang Cui is Professor at the Institute of Nano Biomedicine and Engineering, Shanghai Jiao Tong University, and Editor-in-Chief of Nano Biomedicine and Engineering. His research interests focus on nano theranostic technologies and their clinical translations.

At the end of 2019, novel coronavirus (2019-nCoV) broke out globally. So far, global infection patients have passed 67 million (as on Dec. 8, 2020), the death toll has risen sharply. Especially in the United States, Italy, India and other countries, novel coronavirus epidemics are still not controlled very well, with new cases increasing quickly. 2019-nCoV has become the most serious global public health events within 60 years.

The novel coronavirus structural genome is 29883bp, containing 14 ORF frames. The larger protein molecules include the following protein regions, $\mathrm{N}$ : nucleocapsid protein, S: Spike protein, M: membrane protein, HE: hemagglutinesterase, and E: envelope protein. The infection of cells is mainly through the binding of $S$ protein with ACE2 on the cell surface. It mainly invades human lung tissues, causes pulmonary inflammation and fibrosis, and produces serious complications. Many research groups in the world have been focusing on the development of theranostic technology of 2019-nCoV.
The urgent problems and challenges to be solved are: how to quickly screen out patients with new coronavirus infection and carry out rapid isolation and treatment; how to maintain the cleanliness and disinfection of public places, public transportation, elevators, vehicle environment, hospitals, and home indoor environment, effectively kill new coronavirus and bacteria, and avoid contact transmitted infection; how to develop therapeutic antibodies against new coronavirus; and how to develop new vaccine to prevent new coronavirus infection, and to save the lives of infected persons.

Based on years of international cooperation and early technological accumulation, the team in Shanghai National Engineering Research Center for Nanotechnology rapidly initiated communication and discussion when the epidemic occurred, proposed the research and development plan, raised research and development funds, and quickly started projects to solve the key technical problems of new coronavirus prevention, early rapid detection reagents and 
instruments, therapeutic antibodies and nano-vaccines. Up to date, the team has developed the disinfectants and air cleaners to prevent virus transmission, nucleic acid extraction kit and automatic nucleic acid extractor, nucleic acid detection kit for new coronavirus, colloidal gold and magnetic nanoparticles-labeled chromatography chip for new coronavirus, the equipment and detection kit for aerosol collection of new coronavirus, and therapeutic antibody and nano vaccine against novel coronavirus. The developed disinfectant has obtained FDA certification; the nucleic acid extraction kit and automatic nucleic acid extraction instrument have obtained medical device certifications; and the nucleic acid detection kit and colloidal gold detection kit have obtained EU CE certification. Most of the developed products have been applied in the fight against new coronavirus epidemic.
This special issue focuses on the main progress achieved by the team of National Nano Engineering Research Center and its partners together to develop the rapid detection kit of new coronavirus, disinfectant and air cleaner for preventing the spread of novel coronavirus, and therapeutic antibody and vaccine against the new coronavirus. This special issue bears the aim to promote enterprise cooperation and clinical transformation of new coronavirus diagnosis and therapeutic technology.

Copyright $\subseteq 2020$ Daxiang Cui. This is an open-access article distributed under the terms of the Creative Commons Attribution License, which permits unrestricted use, distribution, and reproduction in any medium, provided the original author and source are credited. 